\title{
A LARGE CLASS OF SMALL VARIETIES OF LATTICE-ORDERED GROUPS
}

\author{
E. B. SCRIMGER 1
}

ABSTRACT. We establish the existence of a countable collection of varieties, each of which covers the abelian variety in the lattice of varieties of lattice-ordered groups.

For each positive integer $n$, let $\mathscr{L}_{n}$ denote the variety of lattice-ordered groups (hereafter, l-groups) satisfying the law $x^{n} y^{n}=y^{n} x^{n}$. Obviously, $\mathfrak{L}_{1}=\mathfrak{L} A$, the variety of abelian $l$-groups, and $\mathfrak{L}_{n}$ is contained in $\mathfrak{L}_{m}$ if $n$ is a divisor of $m$. Martinez [4] notes that the containment $\mathfrak{L}_{n} \subseteq \mathfrak{L}_{m}$ is proper if $n$ is a proper divisor of $m$ by considering a type of example which fails to be commutative or representable in intuitively the least way: the examples are each generated by two noncommuting positive elements $a$ and $x$ such that distinct conjugates of $a$ by powers of $x$ are disjoint. Thus, one is led to suspect that because the examples are barely nonabelian, the varieties they generate might be minimally nonabelian. It will facilitate the discussion to describe these examples as subgroups of a wreath product of ordered permutation groups. (For background, definitions, etc. concerning ordered permutation groups, the reader is referred to [1], [2].)

Let $\mathrm{Z} \backslash \mathrm{Z}$ be the ordered wreath product of two copies of the integers, and define

$$
G_{n}=\left\{((. ., w(z), \ldots), \bar{u}) \in \mathbf{Z} \chi^{\prime} \mathbf{Z} \mid i \equiv j(\bmod n) \Rightarrow w(i)=w(j)\right\} .
$$

Then $G_{n} \in \mathscr{L}_{n}$. Let $a, x$ denote the elements of $G_{n}$ given by:

$$
\begin{gathered}
\bar{a}=0, \quad a(z)= \begin{cases}1 & \text { if } z \equiv 0(\bmod n), \\
0 & \text { if } z \equiv 0(\bmod n),\end{cases} \\
\bar{x}=1, \quad x(z)=0 \quad \forall z \in \mathbf{Z} .
\end{gathered}
$$

Presented to the Society, January 28, 1973; received by the editors August 29, 1973 and, in revised form, June 10, 1974.

AMS (MOS) subject classifications (1970). Primary 06A55; Secondary 08A15.

Key words and phrases. Lattice-ordered group, variety, wreath product.

1 This work was partially supported by a Cottrell College Science grant from the Research Corporation. 
Then $\overline{a^{m} x^{m}}=m=\overline{x^{m} a^{m}}$, and

$$
\begin{aligned}
\left(a^{m} x^{m}\right)(z) & = \begin{cases}m & \text { if } z \equiv 0(\bmod n), \\
0 & \text { if } z \equiv 0(\bmod n),\end{cases} \\
\left(x^{m} a^{m}\right)(z) & =\left(x^{m}\right)(z)+\left(a^{m}\right)(z+m)=\left(a^{m}\right)(z+m) \\
& = \begin{cases}m & \text { if } z+m \equiv 0(\bmod n), \\
0 & \text { if } z+m \equiv 0(\bmod n) .\end{cases}
\end{aligned}
$$

Hence, $x^{m} a^{m}=a^{m} x^{m}$ iff $m \equiv 0(\bmod n)$, iff $n \mid m$. Consequently, if $n$ is a proper divisor of $m$, then $m$ does not divide $n$, so $G_{m} \notin \mathscr{L} n$. It also follows, as Martinez suggests, that if $\mathscr{L}_{n}$ is contained in $\mathfrak{L}_{m}$, then $n$ is a factor of $m$ since

$$
\mathfrak{L}_{n} \subseteq \mathfrak{L}_{m} \Rightarrow G_{n} \in \mathfrak{L}_{m} \Rightarrow a^{m} x^{m}=x^{m} a^{m} \Rightarrow n \mid m \text {. }
$$

It seems apparent that $G_{n}$ is a minimal nonabelian l-group satisfying $x^{n} y^{n}=y^{n} x^{n}$. More precisely, we shall show that if $n$ is prime, the variety of $l$-groups generated by $G_{n}$ is minimal with respect to containing nonabelian members, by showing that every nonabelian, subdirectly irreducible $l$-group in $\mathscr{L}_{n}$ contains an $l$-subgroup $l$-isomorphic to $G_{n}$.

Lemma 1. If $C$ is a convex l-subgroup of $G \in \mathscr{L}_{n}$, then $x^{-n} C x^{n}=C$ for all $x \in G$.

Proof. Suppose $1<c \in C$. Then

$$
1<c<c^{2}<\ldots<c^{n}=x^{-n} c^{n} x^{n} \in x^{-n} C x^{n},
$$

so $c \in x^{-n} C x^{n}$, and hence $C \subseteq x^{-n} C x^{n}$. Similarly, $C \subseteq x^{n} C x^{-n}$, so $C=$ $x^{-n} C x^{n}$

Lemma 2. If $C$ is a convex l-subgroup of $G \in \mathfrak{L}_{n}$ and $x \in G$, then the number of distinct conjugates of $C$ of the form $x^{-i} C x^{i}$ is a divisor of $n$.

Proof. Let $i$ be the smallest positive integer such that $x^{-i} C x^{i}=C$. If $i$ is not a divisor of $n$, then there are integers $r$ and $s$ such that $m+$ $s i=k$, where $1 \leq k<i$, and $k$ is the greatest common divisor of $n$ and $i$. Then $x^{-k} C x^{k}=x^{-r n-s i} C x^{r n+s i}=C$, contradicting the minimality of $i$.

Lemma 3. Let $\mathscr{L} R$ denote the variety of regular l-groups. For any positive integer $n, \mathfrak{L} \cap \mathfrak{L}_{n}=\mathfrak{L} A[4, \$ 6.4]$.

Holland has shown [2] that every $l$-group is $l$-isomorphic to an $l$-subgroup of the l-group of all order-preserving permutations of some chain, 
and that an $l$-group $G$ has a transitive representation as an $l$-group of order-preserving permutations of a chain if and only if $G$ contains a representing subgroup, i.e., a convex prime l-subgroup which contains no nontrivial $l$-ideal of $G$. Among those $l$-groups which have transitive representations are all subdirectly irreducible l-groups. These facts, together with the preceding lemma, yield the following result.

Theorem 1. If $m$ and $n$ are relatively prime, then $\mathfrak{L}_{m} \cap \mathfrak{L}_{n}=\mathfrak{L}$.

Proof. Let $G$ be a subdirectly irreducible member of $\varrho_{m} \cap £ n$, and let $S$ be a chain on which $G$ acts transitively. Suppose $g \in G$ fixes some $x \in S$. Choose integers $r$ and $s$ such that $r m+s n=1$. If $h$ is any member of $G$ such that $x h \neq x$, then

$$
x h=x g^{m n} h^{r m+s n}=x h^{r m} g^{m n} h^{s n}=x h^{r m+s n} g^{m n}=x h g^{m n} .
$$

Therefore, $g$ fixes $x h$, and since $h$ is arbitrary and $G$ is transitive on $S$, $g=1$. Hence, $G_{x}=\{1\}$, so $G$ is totally ordered, and thus regular. But

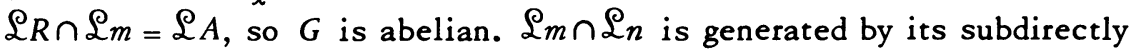
irreducible members, and these are all abelian, so $\mathscr{L}_{m} \cap \mathscr{L}_{n}=\mathfrak{L} A$.

Lemma 4. If $C$ is a representing subgroup of $G \in \mathscr{L}_{n} \backslash \mathfrak{I} A$, then there exists $1<x \in G$ such that $x^{-1} C x \neq C$.

Proof. Suppose $x^{-1} C x=C$ for all $1<x \in G$. Then $C$ is an $l$-ideal of $G$, so since it is also a representing subgroup, $C=\{1\}$, and $G$ is totally ordered, therefore regular. But $\mathscr{L} R \cap \mathfrak{L}_{n}=\mathfrak{L} A$, so $G$ must be abelian. This is a contradiction; therefore, there is a positive $x$ for which $x^{-1} C x \neq$ C.

Lemma 5. If $C$ is a representing subgroup of $G \in \mathfrak{L}_{n}$ which has $n$ dis: tinct conjugates of the form $x^{-i} C x^{i}$ for some $1<x \in G$, then $G$ contains an l-subgroup l-isomorphic to $G_{n}$.

Proof. Suppose we have $G, C, x$ with these properties. We shall find $a_{0} \in G$ such that $a_{0}$ and $x$ correspond under the isomorphism to the two generating elements of $G_{n}$ denoted $a$ and $x$ in the discussion above.

For $0 \leq i \leq n-1$, define

$$
C_{i}=x^{-i} C x^{i}, \quad D_{i}=\bigcap_{j \neq 1} C_{j}
$$

Let $S$ be the chain of right cosets of $C_{0}$, and let $s_{0}$ denote the coset $C_{0}$ in this chain. For $0<i<n-1$, define $s_{i}=s_{0} x^{i}$, so that $C_{i}$ is the stabili- 
zer of $s_{i}$ and $D_{i}$ consists of all permutations of $S$ in $G$ which fix the set $\left\{s_{j} \mid j \neq i\right\}$. Since by hypothesis $C_{0} \neq C_{i}$, there exists a positive permutation $h_{i} \in C_{0} \backslash C_{i}, i=1, \ldots, n-1$, whence

$$
1<g_{0}=h_{1} h_{2} \cdots h_{n-1} \in C_{0} \mid \bigcup_{i=1}^{n-1} C_{i}
$$

Define

$$
d_{0}=x \wedge\left(\bigwedge_{i=1}^{n-1} x^{-i} g_{0}^{n} x^{i}\right)
$$

Since $g_{0}$ fixes only $s_{0}$ among $s_{0}, \ldots, s_{n-1}, x^{-i} g_{0}^{n} x^{i}$ fixes only $s_{i}$ among $s_{0}, \ldots, s_{n-1}$. Thus $d_{0}$ moves only $s_{0}$ among $s_{0}, \ldots, s_{n-1}$ : $1<d_{0} \in D_{0} \backslash \bigcup_{i=1}^{n-1} D_{i}$. Also, $d_{0}<x$ since $x$ moves $s_{0}$. Define $d_{i}=x^{-i} d_{0} x^{i}$, $i=1, \ldots, n-1$. Then $1<d_{i} \in D_{i} \backslash \bigcup_{j \neq i} D_{j}$. By convexity, for any $i$, $0 \leq i \leq n-1$,

$$
e_{i}=\bigvee_{j \neq i}\left(d_{i} \wedge d_{j}\right) \in \bigcap_{k=0}^{n-1} D_{k}=\bigcap_{k=0}^{n-1} C_{k}
$$

Put $a_{i}=x^{-i} a_{0} x^{i}=d_{i} e_{i}^{-1}, i=1, \ldots, n-1$. Then $x^{-n} a_{i} x^{n}=a_{i}, 1<a_{i}<x$, $a_{i} \in D_{i} \backslash \bigcup_{j \neq i} D_{j}, a_{i} \wedge a_{j}=1$ if $i \neq j$, as may be routinely checked. As a consequence of the latter, $a_{i} a_{j}=a_{j} a_{i} \forall i, j$.

For any $u=((\ldots, u(z), \ldots), \bar{u}) \in G_{n}$, define

$$
u \theta:=a_{0}^{u(0)} a_{1}^{u(1)} \cdots a_{n-1}^{u(n-1)} x^{\bar{u}}
$$

It is easily verified that $\theta$ is a group homomorphism.

That $\theta$ is a lattice homomorphism follows from the fact that in $G$,

$$
1 \vee\left(a_{0}^{u(0)} \cdots a_{n-1}^{u(n-1)} x^{\bar{u}}\right)= \begin{cases}1 & \text { if } \bar{u}<0 \\ a_{0}^{u(0) \vee 0} \cdots a_{n-1}^{u(n-1) \vee 0} & \text { if } \bar{u}=0 \\ a_{0}^{u(0)} \cdots a_{n-1}^{u(n-1)} x^{\bar{u}} & \text { if } \bar{u}>0\end{cases}
$$

which we prove as follows. Since $a_{i} \wedge a_{j}=1$ for $i \neq j, \Pi_{i \in T} a_{i}^{a(i)} \wedge$ $\Pi_{i \in T} a_{i}^{\alpha(i)}=1$ where $\alpha(i) \geq 0$ for each $i$ and $T \subseteq\{0,1, \ldots, n-1\}$. Given $a_{0}^{u(0)} \ldots a_{n-1}^{u(n-1)}$, let $T=\{i \mid u(i) \geq 0\}$. Then

$$
\begin{aligned}
\left(a_{0}^{u(0)} \cdots a_{n-1}^{u(n-1)}\right) \vee 1 & =\prod_{i \in T} a_{i}^{u(i)}\left(\prod_{i \neq T} a_{i}^{u(i)} \vee \prod_{i \in T} a_{i}^{-u(i)}\right) \\
& =\prod_{i \in T} a_{i}^{u(i)}\left(\prod_{i \notin T} a_{i}^{-u(i)} \wedge \prod_{i \in T} a_{i}^{u(i)}\right)^{-1} \\
& =\prod_{i \in T} a_{i}^{u(i)}=a_{0}^{u(0) \vee 0} \cdots a_{n-1}^{u(n-1) \vee 0} .
\end{aligned}
$$


Next we show $a_{0}^{m}<x$ for all $m \in \mathbf{Z}$. Since $x>a_{0}>1$, this is so for $m \leq 1$

$$
a_{0}^{m}<x \Leftrightarrow\left(d_{0} e_{0}^{-1}\right)^{m}<x \Leftrightarrow\left(\bigwedge_{j=1}^{n-1}\left(1 \vee d_{0} d_{j}^{-1}\right)\right)^{m}<x \Leftrightarrow\left(1 \vee\left(\bigwedge_{j=1}^{n-1} d_{0} d_{j}^{-1}\right)\right)^{m}<x
$$

so it suffices to show $f^{m} \leq x$ for $m \geq 2$, where $f=\bigwedge_{j=1}^{n-1} d_{0} d_{j}^{-1}$. The induction hypothesis is that $f^{k}<x$ for $0 \leq k<m$. $f^{m}$ is an infimum of terms, one of which is $d_{0} d_{n-1}^{-1} f^{m-2} d_{0} d_{1}^{-1}$. But

$$
d_{0} d_{n-1}^{-1} f^{m-2} d_{0} d_{1}^{-1}=d_{0} x^{1-n} d_{0}^{-1} x^{n-1} f^{m-2} d_{0} x^{-1} d_{0}^{-1} x<x
$$

iff

$$
f^{m-2}<x^{1-n} d_{0} x^{n-1} d_{0}^{-1} x x^{-1} d_{0}^{-1}=x
$$

since $x^{n} d_{0}^{-1}=d_{0}^{-1} x^{n}$. Therefore, $f^{m}<x$, so $a_{0}^{m}<x$ for all $m \in \mathbf{Z}$. By conjugation $a_{i}^{m}<x$ for any $m \in \mathbf{Z}, i=0,1, \ldots, n-1$, so $x^{-1}<a_{0}^{u(0)} \ldots$ $a_{n-1}^{u(n-1)}<x$ for any $u(0), \ldots, u(n-1) \in \mathbf{Z}$, and hence

$$
a_{0}^{u(0)} \cdots a_{n-1}^{u(n-1)} x^{-\bar{u}}<1<a_{0}^{u(0)} \cdots a_{n-1}^{u(n-1)} x^{\bar{u}}
$$

if $\bar{u}>0$, for any $u(0), \ldots, u(n-1) \in \mathbf{Z}$. Thus, (1) holds.

$\theta$ is 1-1 since the element $a$ of $G_{n}$ given by

$$
\vec{a}=0, \quad a(z)= \begin{cases}1 & \text { if } z \equiv 0(\bmod n), \\ 0 & \text { if } z \equiv 0(\bmod n)\end{cases}
$$

belongs to the smallest nontrivial $l$-ideal of $G_{n}$, but $a \theta=a_{0} \neq 1$. This completes the proof of the lemma.

Theorem 2. If $n$ is a prime, the variety of l-groups $\left[G_{n}\right]$.generated by $G_{n}$ covers $\mathcal{L} A$ in the lattice of varieties of l-groups.

Proof. If $G$ is a subdirectly irreducible $l$-group in $\mathcal{L}_{n}$ but not in $\mathfrak{L}_{A}$, then by Lemma 3, $G$ contains a representing subgroup $C$ which is not an $l$-ideal of $G$. Thus, there exists $1<x \in G$ such that $x^{-1} C x \neq C$. Since $n$ is prime, it follows from Lemma 2 that $C$ has $n$ distinct conjugates of the form $x^{-i} C x^{i}$, so by Lemma 5, $G$ contains an $l$-subgroup $l$-isomorphic to $G_{n}$. Therefore, any variety of l-groups contained in $\mathfrak{L}_{n}$ and which has nonabelian members contains the variety $\left[G_{n}\right]$. Hence, $\left[G_{n}\right]$ covers $\mathcal{L} A$ in the lattice of varieties of l-groups if $n$ is prime. 


\section{BIBLIOGRAPHY}

1. P. M. Cohn, Universal algebra, Harper and Row, New York, 1965. MR 31 \# 224.

2. W. C. Holland, The lattice-ordered group of automorphisms of an ordered set, Michigan Math. J. 10 (1963), 399-408.

3. W. C. Holland and S. H. McCleary, Wreath products of ordered permutation groups, Pacific J. Math. 31 (1969), 703-716. MR 41 \#3350.

4. Jorge Martinez, Free products in varieties of lattice-ordered groups, Czechoslovak Math. J. 22 (1972), 535-553.

DEPARTMENT OF MATHEMATICS, SOUTHWESTERN AT MEMPHIS, MEMPHIS, TENNESSEE 38112 